Journal Club

Editor's Note: These short reviews of recent JNeurosci articles, written exclusively by students or postdoctoral fellows, summarize the important findings of the paper and provide additional insight and commentary. If the authors of the highlighted article have written a response to the Journal Club, the response can be found by viewing the Journal Club at www.jneurosci.org. For more information on the format, review process, and purpose of Journal Club articles, please see http://www.jneurosci.org/content/ jneurosci-journal-club.

\title{
Transcranial Direct Current Stimulation Modulates GABA Levels Beyond the Stimulated Region: Perspectives for Stroke Rehabilitation
}

\author{
Takahiro Inoue and Kenji Taneda \\ Graduate School of Health Sciences, Hokkaido University, N12-W5, Kitaku, Sapporo 060-0812, Japan \\ Review of Bachtiar et al.
}

Transcranial direct current stimulation (tDCS) is a method of non-invasive brain stimulation that induces changes in cortical excitability by applying a weak electric current transcranially. The direction of tDCS-induced cortical excitability change depends on the stimulation polarity. In general, anodal tDCS increases and cathodal tDCS decreases motor cortex excitability (Nitsche and Paulus, 2000). In addition to reducing ipsilateral excitability, cathodal tDCS can increase contralateral excitability by decreasing interhemispheric inhibition. (O'Shea et al., 2014). Furthermore, tDCS can also be applied bilaterally. For example, the anode might stimulate one primary motor cortex (M1) while the cathode simultaneously stimulates the contralateral M1 (Vines et al., 2008).

Because tDCS can modulate differently each hemisphere, it might be useful for rehabilitation after stroke, which is a major cause of motor dysfunction. In fact, there is some evidence that tDCS applied either during or before training improved motor function of stroke pa-

Received Aug. 10, 2018; revised Dec. 5, 2018; accepted Dec. 5, 2018. We thank Drs. Hiroshi Maejima and Yasuyuki Takamatsu of Hokkaido University for constructive advice.

The authors declare no competing financial interests.

Correspondence should be addressed to Takahiro Inoue at 11takahir004@gmail.com.

https://doi.org/10.1523/JNEUROSCI.2524-18.2018

Copyright $\odot 2019$ the authors $\quad 0270-6474 / 19 / 391768-03 \$ 15.00 / 0$ tients (Zimerman et al., 2012; Allman et al., 2016). Furthermore, bilateral tDCS (or dual-tDCS) has been proposed for rectifying interhemispheric imbalances after stroke. In the healthy brain, each hemisphere equally inhibits the other via the corpus callosum. However, after stroke, excitability in the ipsilesional hemisphere is reduced. As a result, inhibition from the stroke-affected hemisphere to the contralateral hemisphere is decreased; the contralesional hemisphere thereby becomes more excitable and exerts stronger inhibition on the ipsilesional hemisphere. These excitability imbalances often impair recovery after stroke (Boddington and Reynolds, 2017). Reversing them with bilateral tDCS (placing the anode over the ipsilesional hemisphere, and the cathode over the contralesional hemisphere) might therefore improve recovery.

Despite the theoretical benefits of tDCS in stroke, the latest Cochrane review, a collection of high-quality evidence in the medical field, reports that the evidence that tDCS improves performance of activities of daily in living in stroke patients is inconclusive (Elsner et al., 2016). One possible reason for this conclusion is that interindividual and intra-individual response variability obscures the possible effects of tDCS (López-Alonso et al., 2014, 2015; Guerra et al., 2017). A previous study using a large sample size and cluster analysis reported the presence of "responders" who showed the expected response to tDCS and "non-responders" who did not (López-Alonso et al., 2014). Moreover, López-Alonso et al. (2015) showed that most responders and over half of the non-responders displayed similar responses in the first and second session, indicating that intra-participant variability was lower than inter-participant variability. The sources of this response variability are unclear, but individual anatomical characteristics might be a factor.

In stroke rehabilitation, motor learning beneficially contributes to functional recovery. Recently, it has become apparent that $\mathrm{tDCS}$ applied to M1 affects motor learning, possibly by altering GABA concentrations. In healthy participants, GABA concentration decreases in the M1 during motor learning (Kolasinski et al., 2018), and anodal tDCS can enhance cortical excitability while decreasing GABA concentration in M1 (Stagg et al., 2011a). Furthermore, Stagg et al. (2011a) found a positive correlation between the magnitude of the tDCS-induced GABA decrease in M1 and the degree of motor learning. Therefore, GABA is a candidate biomarker for potential plasticity in response to tDCS, and assessing the effects of various tDCS montages on GABA concentra- 
tion in M1 might help to maximize motor learning.

In a recent report in The Journal of Neuroscience, Bachtiar et al. (2018) examined the effect of anodal, cathodal, and bilateral tDCS on neurochemical changes in M1 using two-voxel magnetic-resonance spectroscopy (MRS). Briefly, current was delivered via electrodes applied over the M1 of 12 healthy participants. For unilateral stimulation, the stimulating electrode was placed over the left M1, and the reference was placed over the right supraorbital ridge. For bilateral stimulation, the anode was placed over the left M1 and the cathode was placed over the right M1. Neurochemical changes, including changes in GABA and glutamate concentrations, were quantified at five time points: before tDCS (baseline), during tDCS, and at three time points after tDCS (Post 1: 1-13 min, Post 2: 14-26 min, and Post 3: 27-39 min after tDCS).

For unilateral stimulation protocols, anodal stimulation decreased GABA concentration in both the stimulated and unstimulated M1, whereas cathodal tDCS decreased GABA concentration only in the unstimulated M1. These tDCS-induced GABA changes lasted for at least $30 \mathrm{~min}$ after stimulation. Glutamate concentration was not affected by stimulation in either M1 with any montage. These results using MRS are generally consistent with those of a previous study using electrophysiological verification, in which the effects of tDCS on motor-evoked potential were examined as an indicator of corticospinal excitability (O'Shea et al., 2014). Thus, these results supported tDCSinduced changes in excitability, based on both electrophysiological changes and neurochemical changes.

For bilateral stimulation, Bachtiar et al. (2018) found that GABA concentration decreased only in the cathodetargeted M1. Although bilateral tDCS is theoretically a sum of anodal and cathodal tDCS, this result indicates that the physiological effects of bilateral stimulation were not simply the sum of the constituent unilateral montages (anodal and cathodal tDCS). One clear reason for this is that current flows differently between the electrodes in the various montages. Specifically, currents are directed in a dorsal to ventral manner in the unilateral montages, whereas they are directed in a lateral to medial manner in the bilateral montage (Wagner et al., 2007). In addition, the pattern of change in network connectivity critically depends on the montage. Sehm et al. (2012) showed that bilateral mon- tage modulated network connectivity in the primary motor, secondary motor, and prefrontal areas, whereas unilateral montage affected prefrontal, parietal, and cerebellar areas; these findings indicated that the modulated networks differed between bilateral and unilateral tDCS. Thus, although Bachtiar et al. (2018) demonstrated that both bilateral and unilateral tDCS decrease M1 GABA levels, this result might have been mediated by different underlying physiological mechanisms operative in bilateral versus unilateral montages. Further studies are needed to investigate the influence of electrode position (particularly with regard to polarity and current flow direction) on the tDCSinduced physiological effect.

Next, Bachtiar et al. (2018) investigated how the GABA reduction seen in the unstimulated hemisphere with unilateral tDCS might relate to the microstructure of white-matter fibers running between the M1s in the corpus callosum (CC). They found that cathodal tDCS-induced GABA change in the unstimulated M1 was negatively correlated with the structural integrity of the M1-to-M1 callosal fibers; specifically, greater structural integrity in the CC was associated with a greater decrease in GABA levels in the unstimulated M1. In contrast, there were no significant correlations between GABA levels in the unstimulated M1 and the integrity of two other fiber tracts: the section of CC connecting the stimulated M1 to the unstimulated somatosensory cortex and the corticospinal tract. Thus, cathodal tDCS-induced GABA changes in the unstimulated M1 depend on the integrity of a specific interhemispheric connection. This might be a significant contributor to the variability in therapeutic results obtained with tDCS. Bachtiar et al. (2018) hypothesized that stroke patients with structurally intact callosal fibers might benefit from cathodal tDCS applied to the contralesional M1.

Regarding future clinical application of tDCS, several animal studies have shown that extrasynaptic GABAergic inhibition in the cortex influences recovery from focal stroke. Clarkson et al. (2010) showed that tonic neuronal inhibition mediated by extrasynaptic $\mathrm{GABA}_{\mathrm{A}}$ receptor in the peri-infarct cortex increased after stroke, and that this increased tonic inhibition worsened functional recovery, likely because the increased tonic inhibition limits the cortical plasticity necessary for recovery. Indeed, reducing extrasynaptic GABAergic inhibition enhanced functional recovery after stroke (Lake et al., 2015; Orfila et al., 2017). Because the MRS-assessed GABA level likely reflects extrasynaptic GABAergic tone (Stagg et al., 2011b) and Bachtiar et al. (2018) showed that all three tDCS montages decrease M1 GABA levels as assessed by MRS, all tDCS montages might enhance functional recovery after stroke by decreasing extrasynaptic GABAergic inhibition.

In conclusion, Bachtiar et al. (2018) revealed the effect of three tDCS montages on neurochemical changes in bilateral $\mathrm{M} 1$, and the relation between neurochemical changes in the unstimulated hemisphere and the microstructure of the white matter connecting bilateral M1s. Furthermore, their study results suggested that structural integrity in the CC could be an indicator of cathodal tDCS-induced GABA changes beyond the stimulated region. These findings increase our understanding of the wider motor network underpinning $\mathrm{tDCS}$-induced motor plasticity and might help to explain the tDCS response variability in stroke rehabilitation.

\section{References}

Allman C, Amadi U, Winkler AM, Wilkins L, Filippini N, Kischka U, Stagg CJ, Johansen-Berg H (2016) Ipsilesional anodal tDCS enhances the functional benefits of rehabilitation in patients after stroke. Sci Transl Med 8:330re1.

Bachtiar V, Johnstone A, Berrington A, Lemke C, Johansen-Berg H, Emir U, Stagg CJ (2018) Modulating regional motor cortical excitability with non-invasive brain stimulation results in neurochemical changes in bilateral motor cortices. J Neurosci 38:7327-7336.

Boddington LJ, Reynolds JNJ (2017) Targeting interhemispheric inhibition with neuromodulation to enhance stroke rehabilitation. Brain Stimul 10:214-222.

Clarkson AN, Huang BS, Macisaac SE, Mody I, Carmichael ST (2010) Reducing excessive GABA-mediated tonic inhibition promotes functional recovery after stroke. Nature 468 : 305-309.

Elsner B, Kugler J, Pohl M, Mehrholz J (2016) Transcranial direct current stimulation (tDCS) for improving activities of daily living, and physical and cognitive functioning, in people after stroke. Cochrane Database Syst Rev 3:CD009645.

Guerra A, López-Alonso V, Cheeran B, Suppa A (2017) Solutions for managing variability in non-invasive brain stimulation studies. Neurosci Lett. Advance online publication. Retrieved December 1, 2018. doi:10.1016/ j.neulet.2017.12.060.

Kolasinski J, Hinson EL, Z APD, Rizov A, Emir UE, Stagg CJ, 1 (2018) The dynamics of cortical GABA in human motor learning. J Physiol. Advance online publication. Retrieved December 1, 2018. doi:10.1113/ JP276626.

Lake EM, Chaudhuri J, Thomason L, Janik R, Ganguly M, Brown M, McLaurin J, Corbett D, Stanisz GJ, Stefanovic B (2015) The effects of delayed reduction of tonic inhibition on isch- 
emic lesion and sensorimotor function. J Cereb Blood Flow Metab 35:1601-1609.

López-Alonso V, Cheeran B, Río-Rodríguez D, Fernández-Del-Olmo M (2014) Inter-individual variability in response to non-invasive brain stimulation paradigms. Brain Stimul 7:372-380.

López-Alonso V, Fernández-Del-Olmo M, Costantini A, Gonzalez-Henriquez JJ, Cheeran B (2015) Intra-individual variability in the response to anodal transcranial direct current stimulation. Clin Neurophysiol 126:2342-2347.

Nitsche MA, Paulus W (2000) Excitability changes induced in the human motor cortex by weak transcranial direct current stimulation. J Physiol 527:633-639.

Orfila JE, Grewal H, Dietz RM, Strnad F, Shimizu T, Moreno M, Schroeder C, Yonchek J, Rodgers KM, Dingman A, Bernard TJ, Quillinan N, Macklin WB, Traystman RJ, Herson PS (2017) Delayed inhibition of tonic inhibition enhances functional recovery following experimental ischemic stroke. J Cereb Blood Flow Metab. Advance online publication. Retrieved December 1, 2018. doi:10.1177/ $0271678 X 17750761$.

O'Shea J, Boudrias MH, Stagg CJ, Bachtiar V, Kischka U, Blicher JU, Johansen-Berg H (2014) Predicting behavioural response to TDCS in chronic motor stroke. Neuroimage 85:924933.

Sehm B, Schäfer A, Kipping J, Margulies D, Conde V, Taubert M, Villringer A, Ragert P (2012) Dynamic modulation of intrinsic functional connectivity by transcranial direct current stimulation. J Neurophysiol 108:3253-3263.

Stagg CJ, Bachtiar V, Johansen-Berg H (2011a) The role of GABA in human motor learning. Curr Biol 21:480-484.

Stagg CJ, Bestmann S, Constantinescu AO, Moreno LM, Allman C, Mekle R, Woolrich M, Near J, Johansen-Berg H, Rothwell JC (2011b) Relationship between physiological measures of excitability and levels of glutamate and GABA in the human motor cortex. J Physiol 589:5845-5855.

Vines BW, Cerruti C, Schlaug G (2008) Dualhemisphere $\mathrm{tDCS}$ facilitates greater improvements for healthy subjects' non-dominant hand compared to uni-hemisphere stimulation. BMC Neurosci 9:103.

Wagner T, Fregni F, Fecteau S, Grodzinsky A, Zahn M, Pascual-Leone A (2007) Transcranial direct current stimulation: a computerbased human model study. Neuroimage 35:1113-1124.

Zimerman M, Heise KF, Hoppe J, Cohen LG, Gerloff C, Hummel FC (2012) Modulation of training by single-session transcranial direct current stimulation to the intact motor cortex enhances motor skill acquisition of the paretic hand. Stroke 43:2185-2191. 